\title{
On the coupling of massless particles to scalar fields
}

\author{
Hartmut Frommert \\ E-Mail: Hartmut.Frommert@uni-konstanz.de \\ Dept. of Physics, University of Constance \\ P.O.Box 5560 M 678, D-78434 Konstanz, Germany
}

August 1, 2018 


\section{Abstract}

It is investigated if massless particles can couple to scalar fields in a special relativistic theory with classical particles. The only possible obvious theory which is invariant under Lorentz transformations and reparametrization of the affine pa-

rameter leads to trivial trajectories (straight lines) for the massless case, and also the investigation of the massless limit of the massive theory shows that there is no influence of the scalar field on the limiting trajectories.

On the other hand, in contrast to this result, it is shown that massive particles are influenced by the scalar field in this theory even in the ultra-relativistic limit. 


\section{Introduction}

In the context of light deflection in gravitational fields, it has been pointed out that within the Newtonian theory of gravity (i.e., gravity as a scalar field), energy conservation arguments lead to effects on light propagation (Soldner 1801). This can be seen more fundamentally by considering classical particles, massive or massless, which propagate with light velocity $c$ through a scalar field acting on them in the absolute Newtonian spacetime.

Going over to the special-relativistic Minkowskian spacetime, the situation gets different, as in this case there occurs a fundamental difference between massive and massless particles, with respect to their propagation. Within this theoretical framework, one can see quite soon that it is no more clear if massless particles can couple to scalar fields at all.

That such a coupling is impossible has been claimed occasionally, but without rigid proof, even in some textbooks such as Greiner 1989. It is also of interest with respect to particle physics, where the Higgs mechanism is used to generate mass by a scalar field, implying that coupling to the scalar Higgs field occurs only for massive particles (more acurately, even to the masses of the particles; this interaction has been studied in detail by Dehnen and Frommert 1990, 1991; Dehnen et.al. 1990). However, on the field theoretical level, it is possible to add coupling to massless particle fields by hand, provided only that the scalar field has a trivial ground 
state, simply such an interaction was not found in nature and, therefore, can be very weak at best.

In order to investigate if massless particles can couple to scalar fields in a special relativistic theory with classical particles, it is most convenient to find a Lagrange function (and thus a theory) which is invariant under Lorentz transformations and reparametrization of the affine parameter which replaces time in special relativistic theories?. As outlined in this work, there is only one possible obvious theory of coupling relativistic particles to scalar fields which matches these requirements. This theory turns out to be trivial for the case of massless particles, i.e. the trajectories of lightlike particles are straight lines. Moreover, the investigation of the massless limit of the more general theory shows that there is no influence of the scalar field on the limiting trajectories. This result reproduces the wellknown fact that because of the observed light deviation in gravitational fields, gravity cannot be described correctly by a special-relativistic scalar theory in flat Minkowskian spacetime.

On the other hand, one may suspect that the problem of massless particles may be correlated in some way with the relativistic limit of the massive problem. This limit is also investigated here. In difference to the result above, it turns out that massive particles are deflected by the scalar field in this theory, even in the

\footnotetext{
${ }^{1}$ If it should turn out that it should be impossible to find such a Lagrange function, one had to select a set of equations of motion by hand, and to face all the problems which arise in such theories.
} 
ultra-relativistic limit, i.e. $V \longrightarrow c$.

\section{Lagrange functions for relativistic particles}

According e.g. to Greiner 1989 (eq. (98)) or Lindner 1994, the special relativistic Lagrange function for a massive, free classical particle (i.e., "test body") is given by光

$$
L=-m_{0} c \sqrt{V_{\mu} V^{\mu}}
$$

which must be inserted into the action principle:

$$
S=S\left(t_{1}, t_{2}\right)=\int_{t_{1}}^{t_{2}} L d t
$$

This action is invariant under both Lorentz or Poincaré transformations, and reparametrization of the "parameter" $t$, as $L d t$ may be rewritten with the proper time $\tau$ as parameter, as $L d t=-m_{0} d \tau$.

From this action follows via Hamilton's variation procedure the (vector-valued) Euler-Lagrange equation:

$$
\frac{d}{d t}\left(\frac{1}{\sqrt{V_{\alpha} V^{\alpha}}} V^{\mu}\right)=0
$$

the equation of motion for a (relativistic) free particle. It may be evaluated to

$$
\ddot{x}^{\mu}-\frac{\dot{x}^{\mu} \dot{x}_{\nu}}{\dot{x}^{\alpha} \dot{x}_{\alpha}} \ddot{x}^{\nu}=\left(\delta_{\nu}^{\mu}-\frac{\dot{x}^{\mu} \dot{x}_{\nu}}{\dot{x}^{\alpha} \dot{x}_{\alpha}}\right) \ddot{x}^{\nu}=0 .
$$

\footnotetext{
${ }^{2} m_{0}$ : rest mass, $c$ : light velocity, $t$ : time, $(\ldots)^{\cdot}=\frac{d}{d t}(\ldots), V^{\mu}: 4$-velocity $\left(V^{\mu}=\dot{x}^{\mu}\right)$. Convention: $\eta_{\mu \nu}=\operatorname{diag}(+---)$
} 
It is remarkable that because of the projector, these are only 3 nontrivial independent components of this equation (scalar equations): Contraction with $V_{\mu}=\dot{x}_{\mu}$ yields identically zero. Equation (3) may also be contracted with $u_{\mu}=V_{\mu} / \sqrt{V_{\beta} V^{\beta}}$ to enhance $u^{\mu} u_{\mu}=$ const $\in\{0, \pm 1\}$.

One may straightforwardly add e.g. electromagnetism (as an external field) by adding an interaction term in the following way:

$$
L=-m_{0} c \sqrt{V_{\mu} V^{\mu}}+q A_{\mu} V^{\mu}
$$

yielding the equations of motion for a massive particle in the electromagnetic field:

$$
\frac{d}{d t}\left(\frac{m_{0} c}{\sqrt{V_{\alpha} V^{\alpha}}} V^{\mu}\right)=-q F_{\nu}^{\mu} V^{\nu}
$$

with the usual electromagnetic field strength $F_{\mu \nu}=A_{\nu, \mu}-A_{\mu, \nu}$. This is, of course, the usual Lorentz force equation for a classical point particle. As $F_{\mu \nu}$ is skew symmetric, contraction with $V^{\mu}$ makes both sides of (6) vanish identically, so we have again only 3 independent nontrivial equations.

We remark here immediately that, if $A_{\mu}$ was the 4-gradient of a scalar field (or that of some functional of arbitrary fields), say $\Phi(\boldsymbol{x})$ the right-hand side would vanish identically because of the antisymmetry of the field strength $F_{\mu \nu}$. Therefore, the coupling of gradients to a particle via a potential energy term in the Lagrange function,

$$
L_{i}=\Phi_{, \mu} V^{\mu}
$$

\footnotetext{
${ }^{3} A_{\mu}$ : Electromagnetic 4-potential, $q$ : Electromagnetic (i.e. electric) charge.
} 
does not contribute to the force on the particle. This may even be seen on a more fundamental level: The term evaluates to a pure "surface" term in the action:

$$
S_{i}=\int_{t_{1}}^{t_{2}} L_{i} d t=\int_{\mathcal{C} ; \boldsymbol{x}\left(t_{1}\right)}^{\boldsymbol{x}\left(t_{2}\right)} \Phi_{, \mu} d x^{\mu}=\Phi\left(\boldsymbol{x}\left(t_{2}\right)\right)-\Phi\left(\boldsymbol{x}\left(t_{1}\right)\right),
$$

which is constant if the standard convention of vanishing variations at $t_{1}$ and $t_{2}$ is obeyed. For this reason the coupling to scalar fields must be done in a different way.

For massless fields, the Lagrange function has to be modified: The rest mass $m_{0}$ must be avoided, because it is identically zero. Therefore, we replace the factor $m_{0} c$, which is a "rest momentum", by another, equivalent constant, namely $\hbar / \bar{\lambda}$, i.e. an inverse characteristic wavelength up to a factor $\hbar$ (for massive particles, the wavelength $\bar{\lambda}$ is the Compton wavelength).

In view of the reparametrization invariance of our theory, which is required if we demand the incorporation of space and time into spacetime, as usual in (special and general) relativity, the coordinate time $t$ can no longer play a unique role in our theory . Therefore, the time $t$ in our action can be replaced by any continuous and monotonic parameters $\lambda$ along the curve: $t \longrightarrow \lambda(t)$. Then the velocity $V^{\mu}=d x^{\mu} / d t$ is replaced by

$$
v^{\mu}=\frac{d x^{\mu}}{d \lambda}
$$

\footnotetext{
${ }^{4}$ This demand is forcing for massless particles, as there is no natural invariant parameter for them, such as the proper time for massive particles.
} 
It will be convenient in the following to choose the parameter $\lambda$ in such a way that

$$
d \lambda=\bar{\lambda} d t, \quad v^{\mu}=\frac{d x^{\mu}}{d \lambda}=\frac{V^{\mu}}{\bar{\lambda}} .
$$

Then the Lagrange function and the action take the form:

$$
L=-\hbar \sqrt{v_{\mu} v^{\mu}}=-\hbar \sqrt{\eta_{\mu \nu} \frac{d x^{\mu}}{d \lambda} \frac{d x^{\nu}}{d \lambda}} ; \quad S=\frac{1}{\lambda} \int_{\lambda_{1}}^{\lambda_{2}} L d \lambda .
$$

\section{Coupling scalar fields to relativistic particles}

As the simplest possibility, i.e. adding to the Langrange function a term

$$
L_{i}=q \Phi_{, \mu} V^{\mu}
$$

fails as outlined above, we must look for other couplings. In order to keep the invariance of the action with respect to (arbitrary) reparametrization, the relation $L_{i} \sim 1 / d \lambda$ must be kept. This leads to a first ansatz

$$
L_{i}=-\hbar f\left(\Phi, \Phi_{, \mu}, \ldots\right) \sqrt{v_{\mu} v^{\mu}}
$$

and thus the total Lagrange function

$$
L=-\hbar F\left(\Phi, \Phi_{, \mu}, \ldots\right) \sqrt{v_{\mu} v^{\mu}}
$$

with $F=1+f$, or (see e.g. Misner, Thorne, Wheeler 1973):

$$
L=-\hbar e^{\phi} \sqrt{v_{\mu} v^{\mu}}
$$


with $\phi=\ln F$. Since the scalar field was in no way fixed otherwise on this level, we may now regard $\phi$ as our new scalar field, or scalar potential. This will be justified later by the analogy of the equation of motion to the Newtonian equation with a scalar potential.

Now we take a closer look on the Lagrange function for classical massless particles. The general one-particle Lagrange function $L\left(x^{\mu}, v^{\mu}\right)$ may be expanded in powers of $v^{\mu}$ :

$$
L=l_{0}+\left(l_{1}\right)_{\mu} v^{\mu}+\left(l_{2}\right)_{\mu \nu} v^{\mu} v^{\nu}+\ldots=\sum_{n=0}^{\infty}\left(l_{n}\right)_{\mu_{1} \ldots \mu_{n}} v^{\mu_{1}} \ldots v^{\mu_{n}}
$$

Reparametrization invariance then requires

$$
\left(l_{n}\right)_{\mu_{1} \ldots \mu_{n}}=\frac{\left(f_{n}\right)_{\mu_{1} \ldots \mu_{n}}\left(x^{\mu}\right)}{\left(v_{\alpha} v^{\alpha}\right)^{(n-1) / 2}}
$$

As masslessness corresponds to the condition $v_{\mu} v^{\mu}=0$, one is led to the conclusion that the power series (12) must stop after $n=1$, because otherwise divergences in $L$ would appear (in the form of zero denominator terms). Then equation (11) is the most general Lagrange density possible for a classical particle interacting with a scalar field可.

\footnotetext{
${ }^{5}$ The arguments given here are certainly valid for straightforwardly formed Lagrange functions such as those discussed here. It is however difficult to find a general decision if it is possible to find, within special relativity, some strange coupling term as a counterexample or not. One might think e.g. of replacing the Minkowski metric under the root factor, $\sqrt{\eta_{\mu \nu} v^{\mu} v^{\nu}}$, by some $g_{\mu \nu}=\eta_{\mu \nu}+h_{\mu \nu}(\Phi)$. This however would lead at least to the limits if not out of Special Relativity.
} 
Variation of the Lagrange function (11) w.r.t. the particle trajectory yields the vector valued equation of motion:

$$
0=e^{\phi} \sqrt{v_{\lambda} v^{\lambda}} \phi_{, \mu}-\frac{d}{d \lambda}\left(\frac{e^{\phi} v_{\mu}}{\sqrt{v_{\lambda} v^{\lambda}}}\right)
$$

which can be rewritten as

$$
0=e^{\phi} \frac{1}{\left(v_{\lambda} v^{\lambda}\right)^{3 / 2}}\left(v_{\kappa} v^{\kappa} \delta_{\mu}^{\nu}-v_{\mu} v^{\nu}\right)\left(v_{\rho} v^{\rho} \phi_{, \nu}-\eta_{\nu \rho} \frac{d^{2} x^{\rho}}{d \lambda^{2}}\right)
$$

As the exponential factor is positive, the rest of the equation must vanish. Now with $v^{\mu}$ a zero vector, we have a zero denominator which may cause difficulties to handle this equation: the numerator must be an "even smaller" zero to fulfill it. This requirement can be fulfilled, because the third and fourth factor of equation (15) simplify for $v_{\mu} v^{\mu} \longrightarrow 0$, so that the equation takes the form

$$
0=v_{\mu} v^{\nu} \eta_{\nu \rho} \frac{d^{2} x^{\rho}}{d \lambda^{2}}=v_{\mu} \frac{1}{2} \frac{d}{d \lambda}\left(v_{\nu} v^{\nu}\right)
$$

which has reduced to the free relativistic equation of motion, and has the solution

$$
v_{\nu} v^{\nu}=\text { const }
$$

This shows that

1. the solution $v_{\nu} v^{\nu}=0$ is stable (massless particles propagate always lightlike)

2. the massless particle is apparently not influenced by the scalar field $\phi$. 
The latter statement can also be seen directly from the last factors of equation (15), which can be resolved to

$$
\left(\eta_{\mu \nu}-\frac{v_{\mu} v_{\nu}}{v_{\kappa} v^{\kappa}}\right) \frac{d^{2} x^{\nu}}{d \lambda^{2}}=\left(\delta_{\mu}^{\nu}-\frac{v_{\mu} v^{\nu}}{v_{\kappa} v^{\kappa}}\right) v_{\rho} v^{\rho} \phi_{, \nu}
$$

where obviously the right hand side (the 4 -force) goes to zero with $v_{\nu} v^{\nu}$. It may even be guessed when looking again at the Lagrange function (11), which vanishes together with $v_{\nu} v^{\nu}$, no matter whatever the behaviour of $\phi$ is.

To provide a better understanding of the situation we encounter here, we perform a more acurate investigation of the massless problem as a certain limit of the massive one. This can be accomplished in two ways:

1. Let the mass $m_{0}$ go to zero, $m_{0} \longrightarrow 0$, while the energy

$$
E=p^{0} c=m_{0} c^{2} / \sqrt{1-(V / c)^{2}}
$$

stays finite ("semi-constant")

2. For fixed $m_{0}$ let the (3-) velocity $V$ approach $c$

\subsection{The zero rest mass limit}

In the first case, we consider the problem with any fixed, small, finite mass $m$, and choose the "affine" parameter $\lambda$ in such a way that $v^{\mu}=d x^{\mu} / d \lambda$ is the 4 -momentum $p^{\mu}$ of the test particle, i.e.

$$
v^{\mu}=\frac{d x^{\mu}}{d \lambda}=p^{\mu}=m_{0} V^{\mu}=m_{0} \frac{d x^{\mu}}{d \tau}, \quad d \lambda=\frac{d \tau}{m_{0}}
$$


Herewith, we have

$$
v^{\mu} v_{\mu}=\left(\frac{d \tau}{d \lambda}\right)^{2}=m_{0}^{2}=\text { const }
$$

so that the equation of motion (14) reads:

$$
\frac{d}{d \lambda}\left(\frac{e^{\phi}}{m_{0}} \frac{d x^{\mu}}{d \lambda}\right)=m_{0} e^{\phi} \phi^{, \mu}
$$

or, multiplied by $m_{0}$ :

$$
\frac{d}{d \lambda}\left(e^{\phi} \frac{d x^{\mu}}{d \lambda}\right)=\frac{d}{d \lambda}\left(e^{\phi} p^{\mu}\right)=m_{0}^{2} e^{\phi} \phi^{, \mu}
$$

Going now into the massless limit, i.e. $m_{0} \longrightarrow 0$, this equation approaches

$$
\frac{d}{d \lambda}\left(e^{\phi} \frac{d x^{\mu}}{d \lambda}\right)=\frac{d}{d \lambda}\left(e^{\phi} p^{\mu}\right) \stackrel{0}{=} 0
$$

where "으" indicates equality in the limit $m_{0} \longrightarrow 0$. Thus, in our limit, the product of the 4-momentum $p^{\mu}$ of the particle with the exponential function $e^{\phi}$ of the potential $\phi$ at the particle's location becomes a constant of motion:

$$
e^{\phi} p^{\mu} \stackrel{0}{=} P^{\mu}=\mathrm{const}=\left.p^{\mu}\right|_{\phi=0} \quad, \quad p^{\mu} \stackrel{0}{=} P^{\mu} e^{-\phi}
$$

Investigating now the zeroth component of the 4-momentum, the energy $E=p^{0}$, we have

$$
E=p^{0} \stackrel{0}{=} P^{0} e^{-\phi}
$$

from which we can obtain an expression for $e^{\phi}$ :

$$
e^{\phi} \stackrel{0}{=} \frac{P^{0}}{p^{0}}=\frac{P^{0}}{E}
$$


which may be inserted into the equations for the spatial components $p^{i}, i=1, \ldots, 3$ :

$$
p^{i} \stackrel{0}{=} P^{i} e^{-\phi}=\frac{E P^{i}}{P^{0}}
$$

Separating the direction $n^{i}$ of the 3 -momentum $p^{i}$ from its length $p$ according to (summation over $i=1 \ldots 3$ implied here)

$$
p^{i}=p n^{i}, \quad p=\sqrt{p^{i} p^{i}}, \quad n^{i} n^{i}=1,
$$

the original equation (24) takes the form

$$
p n^{i} \stackrel{0}{=} P^{i} e^{-\phi}
$$

which enforces on $P^{i}$ a decomposition

$$
P^{i}=P n^{i}, \quad P=\sqrt{P^{i} P^{i}}
$$

with the same $n^{i}$ as above, and the relation for the absolute values

$$
p \stackrel{0}{=} P e^{-\phi}=\frac{E P}{P^{0}}
$$

Thus we have shown that along with the $P^{i}$, the direction vector components $n^{i}$ of the 3-momentum (and thus velocity) are constants of motion in the zero restmass limit, so that the flight direction is not influenced by the scalar field, no matter which field or particle configuration is assumed. 


\subsection{The ultra-relativistic $(V \longrightarrow c)$ limit}

Leaving $m_{0}$ now fixed, we have the equation of motion (14), and can again choose the parameter $\lambda$ so that

$$
d \lambda=\frac{d \tau}{m_{0}}, \quad v^{\mu}=p^{\mu}
$$

as above (19). The resulting equation of motion is again (22):

$$
\frac{d}{d \lambda}\left(e^{\phi} \frac{d x^{\mu}}{d \lambda}\right)=m_{0}^{2} e^{\phi} \phi^{, \mu}
$$

which has again only three independent and one trivial component (this can be seen by contracting with $e^{\phi} p_{\mu}$ and using $\left.p^{\mu} p_{\mu}=m_{0}^{2}\right)$.

Equation (31) can be evaluated to

$$
\frac{d}{d \lambda} p^{\mu}=\left(m_{0}^{2} \eta^{\mu \nu}-p^{\mu} p^{\nu}\right) \phi_{, \nu}=m_{0}^{2} \phi^{, \mu}-p^{\mu} \frac{d}{d \lambda} \phi
$$

For the general case of the scalar field $\phi$, it is at this stage too complicated to investigate the influence on the massive particle's trajectory. However, this is possible for the interesting special case of a static scalar field, i.e. $\partial_{t} \phi=\phi_{, 0}=0$; then we have from (32) for the zeroth component:

$$
\frac{d}{d \lambda} p^{0}=-p^{0} \frac{d}{d \lambda} \phi
$$

or

$$
0=\frac{1}{p^{0}} \frac{d}{d \lambda} p^{0}+\frac{d}{d \lambda} \phi=\frac{d}{d \lambda}\left(\ln \left(p^{0}\right)+\phi\right) .
$$


This equation has the constant integral (the " $m_{0} c$ " denominator by choice)

$$
\ln \left(\frac{p^{0}}{m_{0} c}\right)+\phi=\mathcal{E}=\text { const }
$$

so that

$$
p^{0}=m_{0} c \cdot e^{\mathcal{E}-\phi}
$$

This is the energy conservation law here in its exact form.

As the zeroth component of the 4-momentum $p^{0}$ is generally given by

$$
p^{0}=\frac{m_{0} c}{\sqrt{1-(V / c)^{2}}},
$$

this is equivalent to

$$
\sqrt{1-(V / c)^{2}}=e^{\phi-\mathcal{E}}, \quad V=c \sqrt{1-e^{2(\phi-\mathcal{E})}}
$$

The energy conservation law can thus be rewritten as

$$
V^{2}=c^{2}\left(1-e^{2(\phi-\mathcal{E})}\right)
$$

As $(V / c)^{2}$ must take values in the real interval $[0,1]$ only, the difference $\phi-\mathcal{E}$ must be negative, i.e. in the field-free $(\phi=0)$ case, the energy constant $\mathcal{E}$ is always positive. It takes the values

$$
\begin{aligned}
\mathcal{E}=0 & \longleftrightarrow \\
\mathcal{E}=\infty & \longleftrightarrow V=c
\end{aligned}
$$


In the non-relativistic limit, the constant $\mathcal{E}$ for the field free case corresponds to

$$
\begin{aligned}
\mathcal{E} & =-\frac{1}{2} \ln \left(1-V^{2} / c^{2}\right) \\
& \approx \frac{1}{2} \frac{V^{2}}{c^{2}}=\frac{E_{k i n}}{m_{0} c^{2}}
\end{aligned}
$$

i.e. the quotient of the kinetic energy by the rest energy $m_{0} c^{2}$ (in case of a nonvanishing scalar field $\phi$, this field occurs here as a potential energy divided by the rest energy).

To discuss the influence on trajectories a little deeper, we investigate now the case of a spherically symmetric, static field. As usual, we have motion in a plain (say the equatorial one) and angular momentum conservation, in addition to the energy conservation from above. From $\phi=\phi(r)$ we have now $\phi_{, \mu}=\phi^{\prime} r_{, \mu}$, and therefore for the tangential component of equ. (32):

$$
\frac{d}{d \lambda} p^{\varphi}+\frac{1}{r} \frac{d r}{d \lambda} p^{\varphi}=-p^{\varphi} \frac{d}{d \lambda} \phi
$$

and thus

$$
0=\frac{1}{r p^{\varphi}} \frac{d}{d \lambda}\left(r p^{\varphi}\right)+\frac{d}{d \lambda} \phi=\frac{d}{d \lambda}\left(\ln \left(r p^{\varphi}\right)+\phi\right)
$$

This equation has the constant integral corresponding to the conserved angular momentum:

$$
\ln \left(r p^{\varphi}\right)+\phi=\ln \mathcal{F}=\text { const }
$$

or

$$
\frac{r p^{\varphi}}{\mathcal{F}}=e^{-\phi}
$$


As the $\varphi$-component of the 4 -momentum is given by

$$
p^{\varphi}=\frac{m_{0} r}{\sqrt{1-(V / c)^{2}}} \frac{d \varphi}{d t}
$$

the angular momentum law reads more explicitely

$$
\frac{m_{0}}{\mathcal{F}} r^{2} \frac{d \varphi}{d t}=\sqrt{1-(V / c)^{2}} e^{-\phi}
$$

or by using the energy conservation law (38):

$$
\begin{aligned}
\frac{m_{0}}{\mathcal{F}} r^{2} \frac{d \varphi}{d t} & =e^{-\mathcal{E}}=\text { const } \\
r^{2} \frac{d \varphi}{d t} & =\frac{\mathcal{F}}{m_{0}} e^{-\mathcal{E}}=: \quad \tilde{\mathcal{F}}=\text { const }
\end{aligned}
$$

This equation does no more depend on the scalar field $\phi$ but only on the energy constant $\mathcal{E}$ and the new constant $\mathcal{F}$. If summarized to a new constant $\tilde{\mathcal{F}}$, this new constant resembles essentially the specific angular momentum of Newtonian mechanics.

With these conservation laws on hand, one can eventually solve the equation for the trajectory approximatively. Inserting the usual expression for $V^{2}$ for motion in a plain,

$$
V^{2}=\left(\frac{d r}{d t}\right)^{2}+r^{2}\left(\frac{d \varphi}{d t}\right)^{2}
$$

and the angular momentum law (47) into the energy conservation law (39) one obtains

$$
V^{2}=\left(\frac{d r}{d t}\right)^{2}+\frac{\left(\mathcal{F} / m_{0}\right)^{2} e^{-2 \mathcal{E}}}{r^{2}}=c^{2}\left(1-e^{2 \phi-2 \mathcal{E}}\right)
$$


or

$$
\left(\frac{d r}{d t}\right)^{2}=\frac{\left(\mathcal{F} / m_{0}\right)^{2} e^{-2 \mathcal{E}}}{r^{2}}-c^{2}\left(1-e^{2 \phi-2 \mathcal{E}}\right)
$$

Equation (49) has the side result that for vanishing $\phi$, which can be assumed at spatial infinity for a field which is localized anyhow (e.g., the field of a localized source), the velocity takes a limiting value $v_{\infty}$, the excess velocity, which is given by the relation (compare eq. (39))

$$
v_{\infty}^{2}=c^{2}\left(1-e^{2 \phi_{\infty}-2 \mathcal{E}}\right)=c^{2}\left(1-e^{-2 \mathcal{E}}\right)
$$

(since $\phi_{\infty}$ must be constant, it may be absorbed in $\mathcal{E}$, and thus set to zero).

In order to obtain the trajectory instead of the time dependent motion, the differential $d t$ in equation (50) can be substituted by $d \varphi$ via the angular momentum law, which yields

$$
\frac{d r}{d \varphi}=\frac{d r / d t}{d \varphi / d t}=r^{2} \frac{e^{\mathcal{E}}}{\mathcal{F} / m_{0} c} \frac{d r}{d t}
$$

Substituting $r$ by $u=\left(\mathcal{F} / m_{0} c\right) \cdot 1 / r$, equation (50) takes the form

$$
\left(\frac{d u}{d \varphi}\right)^{2}+u^{2}=e^{2 \mathcal{E}}-e^{2 \phi} .
$$

This equation of motion can be evaluated if the scalar field $\phi$ is specified. For large distances, it is convenient to expand $\phi$ in orders of $1 / r$ or $u$, i.e. (with positive coefficients $\alpha_{1}, \alpha_{2}$ for the attractive case) $)^{6}$ :

$$
\phi=-\left(\alpha_{1} u+\alpha_{2} u^{2}+\cdots\right),
$$

\footnotetext{
${ }^{6}$ Having in mind a model for gravity, one could e.g. set $\phi=-\left(A / r+\beta A^{2} / r^{2}\right), A \simeq \mathrm{GM} / c^{2}$. Then one has $\alpha_{1}=m_{0} c A / \mathcal{F}=c A e^{-\mathcal{E}} / \tilde{\mathcal{F}}, \alpha_{2}=\beta \alpha_{1}^{2}$.
} 


$$
e^{2 \phi}=1-2 \alpha_{1} u+2\left(\alpha_{1}^{2}-\alpha_{2}\right) u^{2}+\cdots
$$

Inserting this relation in (53), this equation reads, expanded up to the order $u^{2}$ :

$$
\left(\frac{d u}{d \varphi}\right)^{2}=\left(e^{2 \mathcal{E}}-1\right)+2 \alpha_{1} u-\left(1+2 \alpha_{1}{ }^{2}-2 \alpha_{2}\right) u^{2}+\mathcal{O}\left(u^{3}\right)
$$

This trajectory equation is solved by the following trajectory, up to the order $u^{2}$ :

$$
u=u_{0}\left[1+\epsilon \cos \left\{a\left(\varphi-\varphi_{0}\right)\right\}\right]
$$

with the arbitrary integration constant $\varphi_{0}$ (which determines the periapsis angle) and

$$
\begin{aligned}
u_{0} & =\frac{\alpha_{1}}{1+2 \alpha_{1}^{2}-2 \alpha_{2}} \\
\epsilon & =\sqrt{1+\frac{1}{\alpha_{1}^{2}}\left(e^{2 \mathcal{E}}-1\right)\left(1+2 \alpha_{1}^{2}-2 \alpha_{2}\right)} \\
a & =\sqrt{1+2 \alpha_{1}^{2}-2 \alpha_{2}}
\end{aligned}
$$

( $u_{0}$ determines the periapsis, or size of the trajectory, $\epsilon$ is the excentricity and determines its shape, while $a$ describes the rotation of the trajectory). The proper trajectory equation eventually reads, with these quantities:

$$
r=\frac{r_{0}(1+\epsilon)}{1+\epsilon \cos \left\{a\left(\varphi-\varphi_{0}\right)\right\}}
$$

with the periapsis $r_{0}=\mathcal{F} /\left[m_{0} c u_{0}(1+\epsilon)\right]$. The expression for $\epsilon$, equation (59), can be reformulated to show that it approaches infinity for increasing $\mathcal{E}$, corresponding to $V \longrightarrow c$ :

$$
\epsilon=e^{\mathcal{E}} \sqrt{2\left[\left(1+\frac{1 / 2-\alpha_{2}}{\alpha_{1}{ }^{2}}\right)-e^{-2 \mathcal{E}}\left(\frac{1}{2}+\frac{1 / 2-\alpha_{2}}{\alpha_{1}{ }^{2}}\right)\right]}
$$




$$
\stackrel{\mathcal{E} \rightarrow \infty}{\longrightarrow} e^{\mathcal{E}} \sqrt{2\left(1+\frac{1 / 2-\alpha_{2}}{\alpha_{1}^{2}}\right)}=e^{\mathcal{E}} \frac{a}{\alpha_{1}} \longrightarrow \infty
$$

This means that the trajectory's form approaches a straight line with increasing $\mathcal{E}$, or as $V$ approaches $c$.

It would be preposterous to conclude from this fact that there is no deflection of the fast massive particle. The asymptotic straight lines for $r \longrightarrow \infty$ are to be calculated as follows: The trajectory goes to asymptotic infinity at the poles of the denominator, i.e. at $\cos \left\{a\left(\varphi-\varphi_{0}\right)\right\}=-1 / \epsilon$, or

$$
\delta \varphi=\left|\varphi-\varphi_{0}\right|=\frac{1}{a} \arccos \left(-\frac{1}{\epsilon}\right)
$$

The deflection angle $\Theta$ is related to $\delta \varphi$ by

$$
\Theta=2 \delta \varphi-\pi
$$

thus

$$
\begin{aligned}
\Theta= & \frac{2}{a} \arccos \left(-\frac{1}{\epsilon}\right)-\pi \\
= & \frac{2}{a}\left(\frac{\pi}{2}+\arcsin \frac{1}{\epsilon}\right)-\pi \\
= & \pi\left(\frac{1}{a}-1\right) \\
& +\frac{2}{a} \arcsin \left[e^{-\mathcal{E}} / \sqrt{2\left\{\left(1+\frac{\alpha_{2}+1 / 2}{\alpha_{1}^{2}}\right)-e^{-2 \mathcal{E}}\left(\frac{1}{2}+\frac{\alpha_{2}+1 / 2}{\alpha_{1}{ }^{2}}\right)\right\}}\right]
\end{aligned}
$$

For the large $\mathcal{E}$ considered, the argument of the arcsin function is small, and thus 
the function $\arcsin x$ is well approximated by $x$. Then the deflection angle goes to

$$
\Theta \approx \pi\left(\frac{1}{a}-1\right)+\frac{e^{-\mathcal{E}}}{a} \sqrt{\frac{2}{\left(1+\frac{\alpha_{2}+1 / 2}{\alpha_{1}{ }^{2}}\right)-e^{-2 \mathcal{E}}\left(\frac{1}{2}+\frac{\alpha_{2}+1 / 2}{\alpha_{1}{ }^{2}}\right)}} \approx \pi\left(\frac{1}{a}-1\right)+\frac{2 \alpha_{1} e^{-\mathcal{E}}}{a^{2}}
$$

and approaches[

$$
\Theta_{c}=\pi\left(\frac{1}{a}-1\right)
$$

for large values of $\mathcal{E}$, corresponding to the limit $V \longrightarrow c$ (compare eq. (40)). This nonvanishing deflection is the result a preceding straight trajectory caused by a trajectory "dragging" of the scalar interaction for massive particles, which is essentially the same effect which can be observed as the periapsis shift for bound elliptical trajectories.

\section{References}

[1] H. Dehnen and H. Frommert. Scalar Gravity and Higgs Potential. International Journal of Theoretical Physics, 29(4):361, 1990.

[2] H. Dehnen and H. Frommert. Higgs field gravity within the Standard Model, International Journal of Theoretical Physics, 30(7):985, 1991.

\footnotetext{
${ }^{7}$ Using the model sketched in footnote 6 , the limiting deflection angle evaluates to

$$
\Theta_{c}=\pi\left(1-1 / \sqrt{1+2 \alpha_{1}^{2}(1-\beta)}\right) \approx \pi \alpha_{1}^{2}(1-\beta)=\pi(1-\beta)\left(m_{0} c A / \mathcal{F}\right)^{2}
$$
}


[3] H. Dehnen, H. Frommert, and F. Ghaboussi. Higgs field gravity. International Journal of Theoretical Physics, 29(6):537, 1990.

[4] Walter Greiner and Johann Rafelski. Spezielle Relativitätstheorie. Theoretische Physik, Vol. 3a. Verlag Harri Deutsch, Thun, 2nd edition 1989.

[5] Albrecht Lindner. Grundkurs theoretische Physik. B.G. Teubner, Stuttgart, 1994. Ch. 3.4.9-10, p. 253.

[6] Charles W. Misner, Kip S. Thorne, and John A. Wheeler. Gravitation. W.H. Freeman and Company, San Francisco, 1973, especially p. 178.

[7] J. Soldner. Über die Ablenkung eines Lichtstrahls von seiner geradlinigen Bewegung durch die Attraktion eines Weltkörpers, an welchem er nahe vorbeigeht. Berliner Astronomisches Jahrbuch 1804, p. 161, 1801. Republished and discussed by P. Lenard, Annalen der Physik, IV. Folge, 65:593, 1921. 